\title{
LASER COOLING: BEYOND ONE FIELD AND ONE DIMENSION
}

\author{
N.P. Bigelow, T. Cai, H. Pu, J. Korn, J. Shaffer \\ Department of 'Physics and Astronomy and the Laboratory for Laser Energetics \\ The University of Rochester, Rochester, NY 14627-0011, USA \\ P.R. HEMmer, D.P. KATZ ${ }^{a}$, AND M.S. ShaIIRIAR ${ }^{b}$ \\ Rome Laboratory / EROP, Ilanscom AFB, MA 01731, USA
}

\begin{abstract}
We describe recent experimental and theoretical results which show that the light pressure force for an atom with more than two levels, in a field with more than one frequency or which is studied in more than one dimension can display striking new features. The importance of these results lies in the fact that they cannot be explained in terms of a two-level, a monochromatic or a one-dimensional theory.
\end{abstract}

PACS numbers: $32.80 . P j$

\section{Introduction}

In recent years, there has been significant interest in the laser manipulation of neutral atoms. This problem has been widely investigated both theoretically and experimentally over the past decade [1]. The most successful of the theoretical efforts have focussed on the specific case of an atom interacting with a one-dimensional optical field. Theoretical progress [2-4] has included. both the prediction of new effects [5] and explanation of experimental observations [6]. For more than one spatial dimension or for an atom interacting with. more than one laser field, however, the picture remains incomplete. This limitation is particularly significant given that most existing beam experiments and all optical trapping experiments are inherently multi-dimensional multi-laser frequency systems. Indeed, many intriguing phenomena have been observed which are unique to multi-dimensional experiments and which are not well understood. In this paper we present recent results in our group and review selected work from other laboratories which seeks to generalize the light pressure force to multiple dimensions, multiple field frequencies and multiple atomic levels. We restrict ourselves to the

Permenant addresses: ${ }^{a}$ IIarvard Univ., Cambridge, MA 02139, USA, ${ }^{b}$ Research Laboratory of Electronics, MIT, Cambridge, MA 02139, USA. 
work most closely related to our own investigations and apologize in advance for neglecting the many other important results which we may not have included.

We begin this paper with a short summary of the light pressure force for a two-level atom interacting with one-dimensional nearly resonant laser field. The successive sections consider the effect of extensions of this theory to more than one dimension, more than one field frequency or more than the two-level atom (TLA). In each case we stress the point that there are significant qualitative changes in the force which are uniquely due to the added dimension, laser field frequency or atomic level.

\section{The force on a two-level atom in a monochromatic one-dimensional laser field: a brief review}

In most situations the force experienced by a two-level atom interacting with a monochromatic light field can be expressed as $F=F_{\mathrm{sp}}+F_{\mathrm{st}}$, where the first and second terms reflect the spontaneous and stimulated components of the force, respectively* [7].

\subsection{Spontaneous forces}

The spontaneous force is the workhorse of many laser cooling experiments and so-called optical molasses. $F_{\text {sp }}$ can be understood in terms of the momentum transferred to an atom as photons are absorbed and reemitted spontaneously. When an atom absorbs a photon it receives a momentum kick and recoils along the wave vector $\boldsymbol{k}$ of the absorbed photon. If the subsequent reemission is via spontaneous emission, then the emission direction is spatially isotropic. Averaged over many events, the momentum transfer to the atom on emission averages to zero, whereas the momentum transfer on absorption is cumulative, producing a finite average force along $k$. The size of this force is of the order of the recoil momentum per absorption $(\hbar k)$ multiplied by the transfer rate $\Gamma$ (where $\Gamma^{-1}$ is the two-level excited state lifetime). For sodium, this corresponds to about $10-20 \mathrm{~N}$, or an acceleration of $\approx 10^{6} \mathrm{~m} / \mathrm{s}$ ! Note that the length scale over which $F_{\text {sp }}$ varies is determined by the variations in $k$ (particularly its direction) and by the spatial variations in the field intensity.

For an atom at rest, the spontaneous light pressure force on the two-level atom can be expressed as $F_{\mathrm{sp}}(r) \approx\left(\rho_{\mathrm{gg}} \sigma_{\mathrm{abs}}-\rho_{\mathrm{ee}} \sigma_{\mathrm{emis}}\right) W(r)$. Here $\rho_{\mathrm{gg}}$ and $\rho_{\mathrm{ee}}$ are the ground and excited state populations, respectively, and the $\sigma$ 's are the absorption and emission cross-sections. The interpretation is straight forward: $\boldsymbol{F}_{\mathrm{sp}}$ is given by the momentum flux at the alomic position, $W(r)$, multiplied by the weighted difference of the ground and excited state populations. Notice that for a stationary atom in an optical standing wave (SW), the net average spontaneous force on the atom is zero since in a SW $W=0$ (the momentum flux associated with each of the counter-propagating traveling waves (TW) which together form the standing wave exactly cancels). From this point of view, for an atom at rest

*A beautiful exposition of these ideas was presented by C. Cohen-Tannoudji in a series of courses presented at the College de France, 1982. 
there is only a net spontaneous force (if the field has effective traveling wave character at the atomic coordinale).

If the light field is an optical standing wave, and the field frequency is detuned below (to the red of) the two-level transition frequency $\omega_{0}$ then spontaneous processes can give rise to a damping force (a cooling force) for finite atomic velocities. The velocity damping can be understood by realizing that for a finite velocity atom the atom will be Doppler shifted closer to resonance with respect to the counterpropagating TW and further from resonance with respect to the co-propagating TW. As a result, the atom will absorb more photons with wave vectors opposite to the velocity than along the velocity. The atom will therefore feel a net average spontaneous force which opposes the motion. For moderate velocities, the size of the force will depend linearly on velocity, justifying the name given this field configuration: optical molasses.

\subsection{Stimulaled forces}

Stimulated forces arise from the interaction between the in-phase component of the dipole moment induced in the atom by the field and the gradient of the field itself. Unlike $F_{\mathrm{sp}}, F_{\mathrm{st}}$ is a conservative force and is often referred to as the dipole or gradient force because it changes sign with detuning and because it depends on the gradient of the field intensity. Because the dipole force is conservative, it can be expressed in terms of a potential [8] $U(x)=-\hbar \Delta \ln [1+p(x)]$, where $p(x)=\left[I(x) / I_{\text {sat }}\right](\beta)^{2} /\left(\Delta^{2}+\beta^{2}\right)$. Ilere $I_{\text {sat }}$ is the two-level saturation intensity, $\Delta=\omega_{\text {laser }}-\omega_{0}$ and $\beta=\Gamma / 2$. IIence, for moderate field intensities or large detunings (e.g. $I<I_{\text {sat }}$ and $\Delta>\beta, g$, where $g$ is the Rabi frequency $2 \mu E / \hbar$ ) we find $F \propto \nabla I(x)$.

More strictly the dipole force results from the momentum transfer associated with the atom mediated redistribution of photons between the TWs which together interfere to produce the SW. This view of the dipole force is most elegantly described in terms of the dressed state. In this picture, the dipole force is derived by taking the gradient of the position dependent dressed state energies, neglecting spontaneous emission.

\section{The dipole force on a TLA in a bichromatic SW light field: rectification and the experiments of Grimm et al.}

In a beautiful series of atomic beam deflection experiments Grimm et al. [9] demonstrated one important effect of having two-laser fields of different frequency simultaneously interacting with a two-level atom: the rectified force. The idea of rectification is that, averaged over an optical wavelength, there can be a finite average value of the dipole force, even at zero velocity. When two-laser fields are simultaneously interacting with the same atom, the possibility of a rectified force arises naturally through the nonlinearity of the atom-field interaction - a long wavelength component of the force can occur because the nonlinear interaction introduces force terms which vary with the beat wa velength of the two-laser fields. The task then is to create a system where this force term is large. 
In the experiments of Grimm et al. the rectification which was observed was described in terms of a spatial modulation of the detuning of one of the SW laser fields due to the other SW field. This description was particularly well suited to their experiments because even though one field had a large Rabi frequency, it was detuned far from resonance $\left(\Delta^{\prime} \approx-300 \Gamma\right)$. The important role of this $\mathrm{SW}$ field was to produce a spatially dependent stark shift of the two-level splitting. By comparison the other SW field was tuned closer to resonance $(\Delta \approx 10 \Gamma)$ and oppositely detuned so that the far-off resonance field created a spatially dependent detuning of the near-resonance field. The effective detuning was given as $\Delta(x)=$ $\Delta+\Gamma^{2}\left[I(x) / I_{\text {sat }}\right] / \Delta^{\prime}$, where $\Delta$ is the detuning of the field closer to resonance and $\Delta^{\prime}$ is the detuning of the far-off resonance field. In a simplified picture, we therefore imagine the near-resonance field as "producing" the force, and the second field as modulating this force. In this model, over certain regions of space, the relative phases between the two fields are such that $\Delta(x)$ flips sign every quarter optical wavelength, just when the gradient in intensity is also flipping sign, thereby causing the dipole force to remain positive definite. Averaged over several optical wavelengths, the force has a non-zero value and is therefore rectified. As noted above, this effect is uniquely associated with the nonlinearity of the atom-field interaction and cannot be explained in terms of a net force which is derived from a superposition of the force associated with the two individual SW ficlds.

This demonstration of a rectified force was striking and confirmed the theoretical ideas of Kazantsev et al. [10], Voitsekhovich et al. [11], and Javanainen [12]. Moreover, the experimental evidence of reclification renewed possibilities for constructing a deep optical trap from such a unidirectional dipole force. [10, 12-13]. These experiments also made an important conceptual point: the two-color force is not necessarily the sum of the two one-color forces.

\section{The force on a TLA in a monochromatic 2D laser field: vortices and anisotropic forces}

There have been several efforts to analyze the light pressure force for more than one dimension. Particular attention has been paid to the case of a two-level atom (TLA) interacting with a two-dimensional (2D) light field. In 1989 Kazantsev and Krasanov [10] pointed out that such an atom can experience a spontaneous light pressure force which can have a vortex texture. This result is somewhat surprising because there is no net averaged momentum flow into or out of the two-dimensional field so that one might expect that $W=0$ and hence that $F_{\mathrm{sp}}=0$. For certain relative phases of the two fields, however, there can be a local traveling wave character in the field. It is this local character of the field which is responsible for the vortical character of the force. Such a field is shown in Fig. 1 which displays $W(r)$. Recently, Hemmerich and Hänsch [14] have reported some experimental observations related to these wavelength scale radiation pressure vortices. Their experiments considered the atomic motion in two crossed, nearly resonant optical standing waves. The experiments, and the theoretical model used to explain the observations, confirmed that the atomic motion (and so the light pressure force) depends strongly on the time-phase delay $\phi$ between the two orthogonal SW fields. 


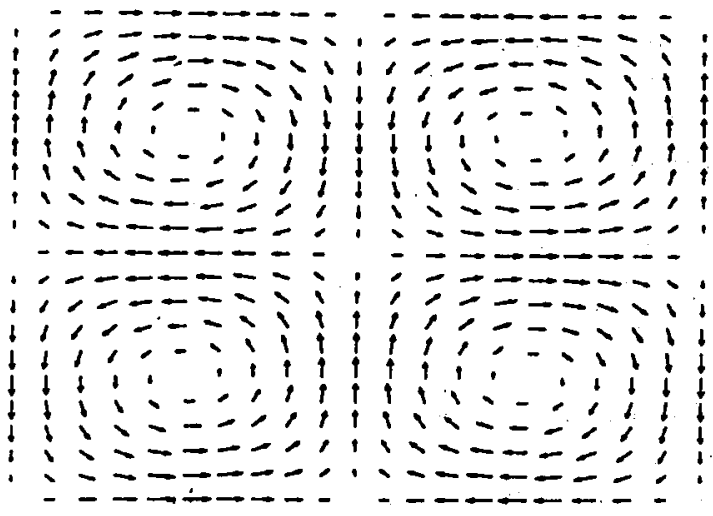

Fig. 1. The momentum flow $W(r)$ as a function of position for a pair of orthogonal optical standing waves of identical frequency and relative phase of $\pi / 2$. Each vortex cell is one-half an optical wavelength in extent.

Throughout both the theoretical and experimental investigations, however, the velocity dependence of the force was not included.

Molmer et al. [15] have recently described their theoretical studies of the spatially-averaged force for both two-level and multi-level atoms interacting with two-dimensional light fields. These workers also predicted a strong dependence of the force on $\phi$, an effect which was confirmed through Monte-Carlo studies. To interpret their results, a dressed state model was introduced which suggested that there might be an anisotropic character to the velocity dependent force.

Recently, we have calculated the spatially averaged force $\boldsymbol{F}(v)$ from the steady-state optical Bloch equations (OBEs) in the low velocity limit by following the treatment of Gordon and Ashkin [7]. We find

$$
F(v)=F_{\text {iso }}(v)+F_{\text {anios }}(v)
$$

where

$$
\begin{aligned}
& \boldsymbol{F}_{\text {iso }}(\boldsymbol{v})=-\alpha_{\mathrm{i}} \hbar k^{2}\left(v_{x} \hat{x}+\mathrm{v}_{y} \hat{y}\right), \\
& \boldsymbol{F}_{\text {aniso }}(\boldsymbol{v})=-\alpha_{\mathrm{a}} \hbar k^{2}\left(v_{x} \hat{x}-v_{y} \hat{y}\right)
\end{aligned}
$$

and

$$
\alpha_{\mathrm{i}}=\frac{1}{2} \frac{\beta \Delta}{\left(\beta^{2}+\Delta^{2}\right)^{2}} g_{0}^{2}\left[-1+\frac{3}{8} \frac{g_{0}^{2}}{\beta^{2}+\Delta^{2}}+\frac{1}{4} \frac{(2+\cos 2 \phi) g_{0}^{2}}{\beta^{2}+\Delta^{2}}\right]
$$

and

$$
\alpha_{\mathrm{a}}=\frac{1}{8} \frac{\beta^{2} g_{0}^{4}}{\left(\beta^{2}+\Delta^{2}\right)^{3}} \sin 2 \phi .
$$

$F(v)$ is composed of an isotropic force contribution (the first term) and an anisotropic spontaneous vortical force contribution (the second term). The first term is isotropic in velocity space and will locally be a cooling or heating force depending on the sign of the detuning $\Delta$ and the local intensity profile of the field. In the low intensity limit, and when the laser is red detuned $(\Delta<0)$, the 
isotropic term will be a cooling or damping force for the atoms similar to that of one-dimensional optical molasses. We note that $\boldsymbol{F}_{\text {iso }}$ is proportional to the detuning $\Delta$, and it will vanish for $\Delta=0$ as well as for $v=0$.

The most surprising contribution to $F(v)$ is $F_{\text {aniso }}(v)$, the spontaneous vortical force. This is the contribution which is anisotropic in velocity space: an atom will experience a cooling force when it moves along one direction while it will experience a heating force when it moves along the perpendicular direction. This force contribution also reflects the local traveling wave character of the field, as shown in Fig. 1.

In the large detuning limit, $\Delta \gg \Gamma$ the isotropic force term will be dominant, and the effect of the anisotropic spontaneous force can be neglected. However as $\Delta$ decreases, the size of the isotropic force also decreases, and the spontaneous vortical force increases. Notice that the anisotropic force has a nonzero value even when $\Delta=0$. If we compare the coefficients of the two terms, we can see that when $|\Delta| \ll\left(g_{0}^{2} / 4 \beta\right)|\sin 2 \phi|$ the anisotropic term will begin to dominate $F(v)$. We also notice that in the lowest order of $g_{0}$ the anisotropic term will increase much faster than the isotropic force as the intensities of the laser fields $\left(g_{0}\right)$ increases.

Following the theme of this paper, we point out that the isotropic force is equivalent to the force that would be predicted by treating the two-dimensional problem as a superposition of two one-dimensional problems. By contrast, the anisotropic term is uniquely associated with the mixing of the two orthogonal spatial dimensions. Experiments are currently underway in our laboratory to verify the presence of this anisotropic spontaneous vortex force, and preliminary results are consistent with our calculations.

\section{The force on a three-level atom in a Raman resonant standing wave: rectification, cooling and dark states}

The interaction between two laser fields and a three-level atom in the $A$ configuration (see Fig. 2) has been of considerable interest for many ycars, wilh the role of ground state coherences playing a central role in this activity. In laser cooling, the interaction of such an atom with two counter propagating traveling

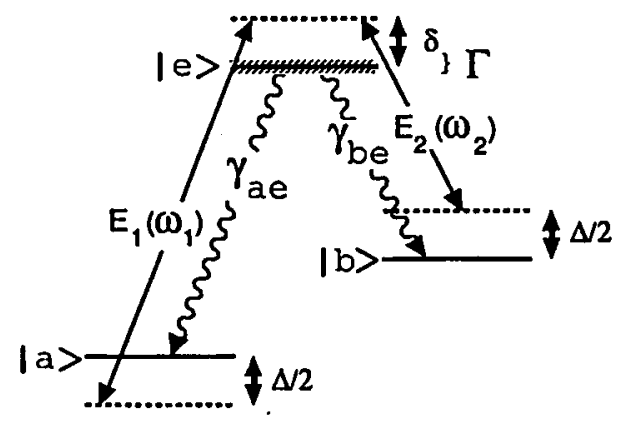

Fig. 2. The three-level $\Lambda$ system. Each leg of the $\Lambda$ is coupled to one field. 
waves (each field driving one of the legs of the $A$ ) has already been used to select atoms [16] with a velocity spread below the single photon recoil, an effect which relied on velocity selective pumping of the population into a dark state [17]. This important effect is known as velocity selective coherent population trapping (VSCPT). More recently, our group [18, 19] and others [20] have solved the steady state optical Bloch equations for an atom interacting with two SW light fields and shown that the net spatially averaged force $\boldsymbol{F}$ on a stationary atom shows features that vary on a length scale both longer and shorter than the optical wavelength (see Fig. 3) [18, 19]. Simplistically, the presence of these long and short wavelength

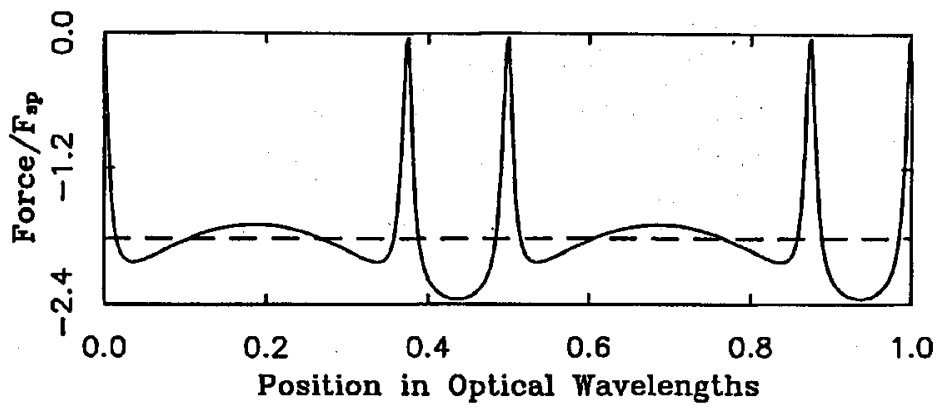

Fig. 3. An example of the rectified force for a $\Lambda$ atom in a one-dimensional Raman standing wave light field. Here $\delta=0, \Delta=g_{0} / 2=4 \Gamma$, the relative phase is $\pi / 4$ and the force is in units of the maximum spontaneous force (from Ref. [21]).

force components also reflects the sum and difference wave vectors of the two SW fields. To describe the components which are longer than the optical wavelength (the rectified components) it is convenient to introduce the phase $\phi=\left(k_{1}-k_{2}\right) x$ between the two standing waves (wave vectors $k_{1}$ and $k_{2}$ ). We find then that $\boldsymbol{F}=0$ when $\phi$ is an integer multiple of $\pi / 2$ and is antisymmetric (in $\phi$ ) with respect to these force zeros. For certain field conditions, the force is simply proportional to $\sin (2 \phi)$.

An intuitive understanding of the force has been provided by calculating the force not in terms of the bare atomic states, but instead in terms of a dark state / bright state basis. This choice of basis is more natural in that it highlights the role of ground state coherences and coherent population trapping in determining the spatially averaged force $[18,19]$.

We have also measured this force experimentally by deflecting an atomic sodium beam [21]. In these experiments one of the SW laser fields was nearly resonant with a transitions from the $F=1$ hyperfine ground state to the excited state and the other SW was nearly resonant with a transition from the $F=2$ ground state. The deflections for several choices of $\phi$ are shown in Fig. 4 where we display the transverse beam profile as a function of position. The details of these experiments are presented in Ref. [21]. We have also carried out experiments and calculations which show that the velocity dependent Raman force can produce efficient cooling [22, 23]. In fact, at low velocities, the cooling is much stronger than 


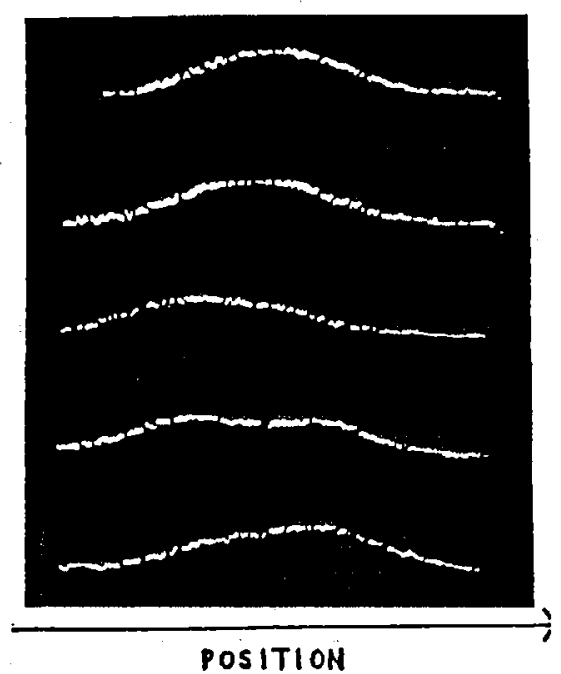

Fig. 4. Deflection data for experiments described in Sec. 5. The top trace is a profile of the atomic beam in the absence of the Raman SW. In descending order, the traces are taken with the Raman field at relative field phases of $0, \pi / 4, \pi / 2$ and $3 \pi / 4$, respectively. Details of this experiment are presented in Ref. [21].

would be expected from a model where the ground state coherences are neglected. This Raman damping depends on $\phi$, an effect which distinguishes it from the corresponding two-level molasses cooling forces.

In sum then, both the strong low velocity cooling and the phase-dependent rectified forces are observed in the interaction of a three-level atom with a Raman SW field. These effects depend on the mixing [12] of the two SW fields in the atom-field interaction and cannot be explained in a simple two-level-like model. The prediction and observation of the cooling is of particular interest because it can be used to greatly enhance the capture rate of atoms by the "zero" velocity trapped state [23]. Experiments are currently under way in our laboratory to build a novel three-dimensional dark-state trap which takes advantage of this process. Finally, we note that as interest in phenomena related to multi-level coherences grows (such as lasing without inversion and self-induced transparency), the understanding of the light pressure force in these three-level systems may become increasingly important.

\section{The force on a three-level atom in a two-dimensional polychromatic laser field: a glimpse at the connection of stimulated rectification and vortices}

Whereas the spontaneous vortex force results directly from the local traveling wave character of the field, we have found that there is a stimulated vortex force experienced by the three-level $\Lambda$ atom in a two-dimensional SW light field. Although qualitatively similar to the two-level heating / cooling spontaneous vortex 


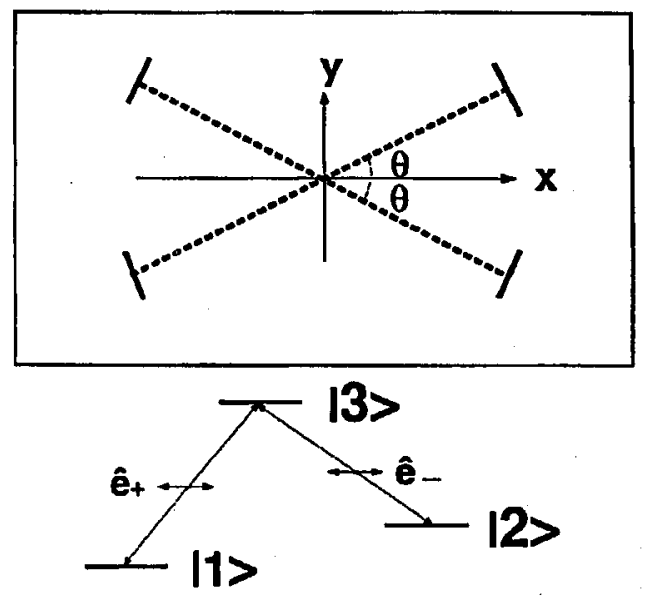

Fig. 5. Field and atomic configuration for Sec. 6.

force, this force has a different origin. This vortex force results from the coherent redistribution of photons between the two standing wave light fields and is present only when the SWs are oriented in different spatial directions (see Fig. 5).

As in Sec. 5, we consider a three-level atom whose transitions are driven by a pair of standing wave light fields. Each field is linearly polarized, with frequencies $\omega_{ \pm}$, respectively and whose polarizations are perpendicular. For clarity we restrict ourselves to the case where the two ground states are degenerate, however we note that the conclusions are valid even in the non-degenerate case. The detunings $\Delta$ and $\delta$ are given as, $\Delta=\Delta_{+}-\Delta_{-}, \delta=\frac{1}{2}\left(\Delta_{+}+\Delta_{-}\right)$and we assume $\delta=0$. The Rabi frequencies $g_{+}=g_{1} \sin \left[\left(k x \cos \theta+\chi_{+}\right)+\left(k y \sin \theta+\chi_{-}\right)\right]$and $g_{-}=$ $g_{2} \sin \left[\left(k x \cos \theta+\chi_{+}\right)-\left(k y \sin \theta+\chi_{-}\right)\right]$and we assume that $|\Delta| \gg\left|g_{ \pm}\right|$. Here $\Delta_{ \pm}=\omega_{ \pm}-\omega_{0}$ and $\omega_{0}$ is the resonant frequency of the transitions. $g_{1}$ and $g_{2}$ are also assumed real. Note that the $\chi$ 's serve only to define the origin in space.

In this case the spontaneous force vanishes, and only a dipole force remains. The zero-velocity force can be found from the density matrix elements as $\boldsymbol{F}=$ $-\left[\rho_{11} \nabla E_{+}+\rho_{22} \nabla E_{-}\right]$, where $E_{ \pm}= \pm(\hbar \Delta / 4) /\left[\beta^{2}+\Delta^{4} / 4\right] g_{ \pm}^{2} \equiv \pm \kappa g_{ \pm}^{2}$. The density matrix elements can be found from the optical Bloch equations $\dot{\rho}_{11}=-\rho_{11} \Gamma_{+}+$ $\rho_{22} \Gamma_{-}$and $\dot{\rho}_{22}=-\rho_{22} \Gamma_{-}+\rho_{11} \Gamma_{+}$, where $\Gamma_{ \pm}=(\beta / 8) g_{ \pm}^{2} /\left(\beta^{2}+\Delta^{2} / 4\right)$. In this limit we find

$$
\boldsymbol{F}=\kappa \frac{g_{+}^{2} \nabla g_{-}^{2}-g_{-}^{2} \nabla g_{+}^{2}}{g_{+}^{2}+g_{-}^{2}}
$$

For compactness we define $\Omega=-g_{1}^{2} g_{2}^{2} /\left(g_{+}^{2}+g_{-}^{2}\right)$ and $\Sigma=\left[\cos 2\left(k x \cos \theta+\chi_{+}\right)-\right.$ $\left.\cos 2\left(k y \sin \theta+\chi_{-}\right)\right]$then

$$
\boldsymbol{F}=\kappa \Omega \Sigma\left[\sin 2\left(k y \sin \theta+\chi_{-}\right) k \cos \theta \hat{x}-\sin 2\left(k x \cos \theta+\chi_{+}\right) k \sin \theta \hat{y}\right]
$$

or

$$
\boldsymbol{F}=-\kappa \Omega \Sigma \frac{1}{2}\left[\nabla \times\left[\cot \theta \cos 2\left(k y \sin \theta+\chi_{-}\right) \hat{z}+\tan \theta \cos 2\left(k x \cos \theta+\chi_{+}\right) \hat{z}\right],\right.
$$

a form which clearly reveals the vortical nature of the force. 

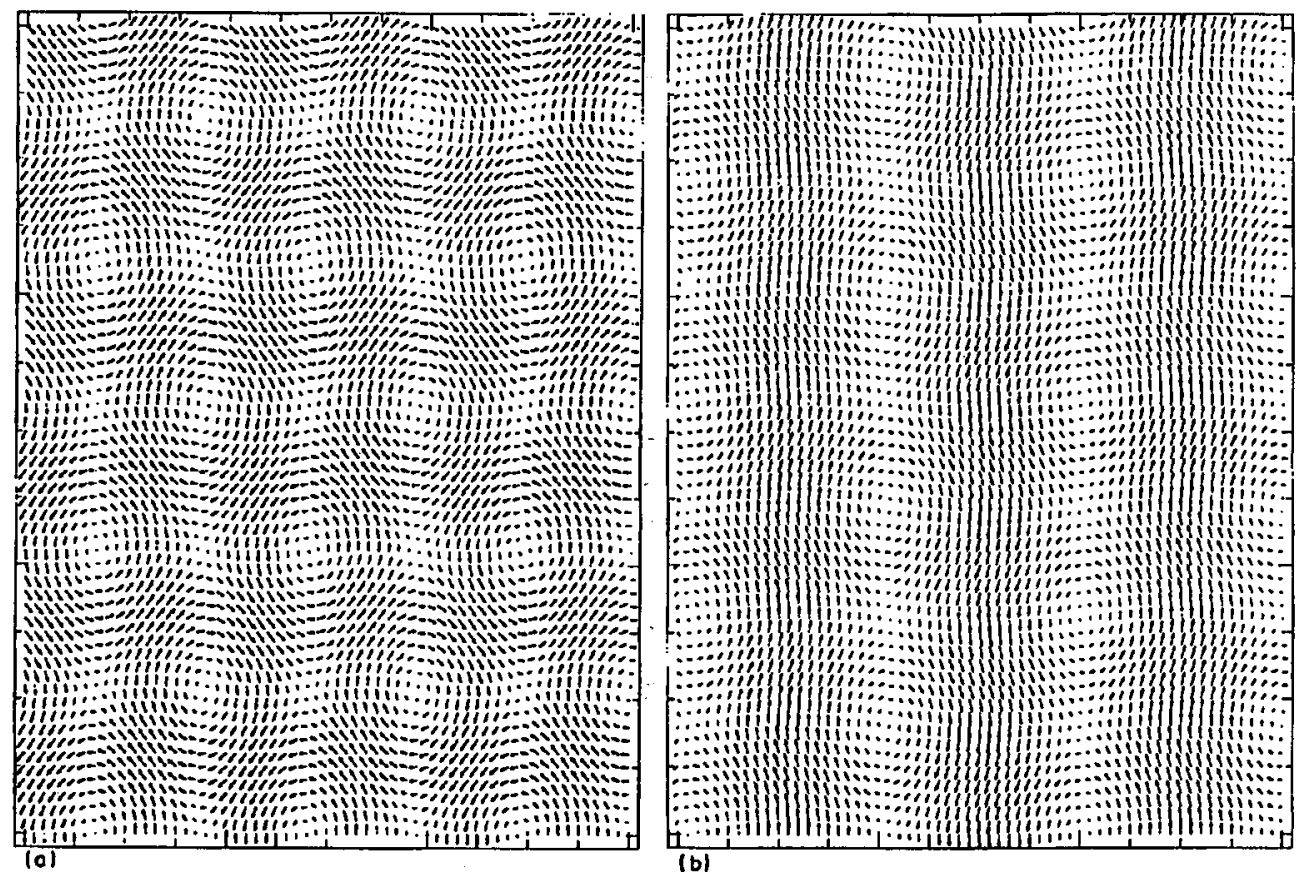

(b)

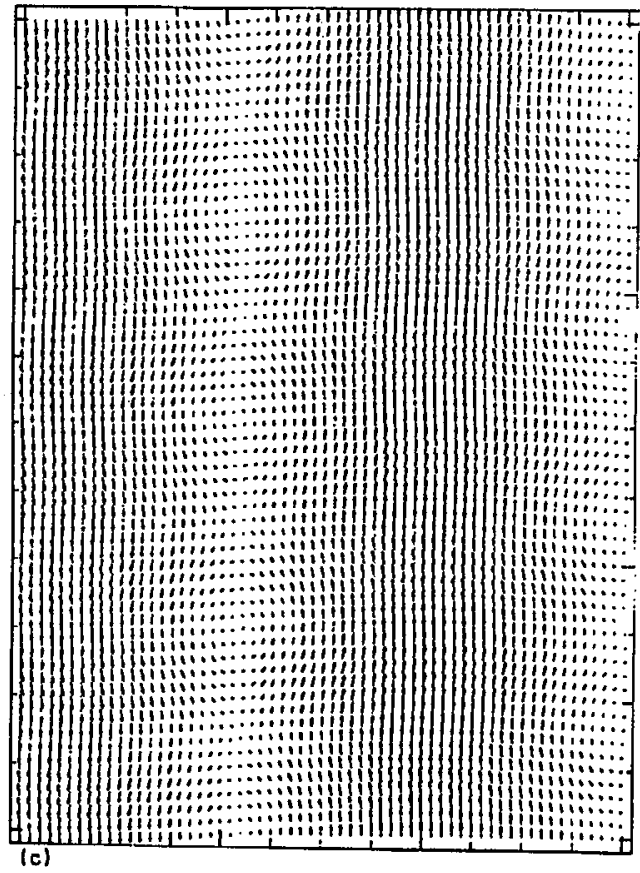

Fig. 6. Vector field plots of the light pressure force discussed in Sec. 6 for different angles $\theta$. (a) $\theta=45^{\circ}$, (b) $\theta=22.5^{\circ}$ and (c) $\theta=11.25^{\circ}$. Notice that as $\theta$ decreases, the force field along the $x$ direction displays a long wavelength character for certain $y$ values. This is the appearance of a rectified force. At $\theta=0$ complete rectification occurs (see text). 
We have also solved for the finite velocity force and find a similar anisotropic component to that found for the two-level atom. We stress, however, that the origin of this vortical force is not at all related to the spontaneous force associated with the local traveling wave character of the light field (as was the case for the two-level two-dimensional vortex force) but instead results from the stimulated redistribution of photons between the two SW laser fields. In fact, for the field geometry considered here, if the ground states are non-degenerate, then the vortical character of $\boldsymbol{W}$ disappears, since the two fields are of different frequency. Ilere it is because the SW fields lie along different spatial directions that there is a vortical contribution to the stimulated force. From this point of view, it is logical to ask whether there is any connection between the stimulated vortex force described here $(\theta \neq 0)$ and the rectified dipole force described in Sec. 5 .

If we consider Eq. (8) in the limit where $\theta \rightarrow 0$, then $\chi_{+} \rightarrow 0$ and $\chi_{-} \rightarrow \chi_{0} / 2$. The force expression call then be rewritten as

$$
\boldsymbol{F}=\kappa \Omega\left[\cos 2 k x-\cos \chi_{0}\right] \sin \chi_{0} k \hat{x}
$$

or

$$
\boldsymbol{F}=\kappa \Omega k\left[\cos (2 k x) \sin \chi_{0}-\frac{1}{2} \sin 2 \chi_{0}\right] \hat{x} .
$$

This expression is identically the expression derived by us earlier [18] for the rectified dipole force in the one-dimensional Raman standing wave light field. Figure 6 shows two-dimensional plots of the light pressure force field for different values of $\theta$. As $\theta$ decreascs, the force field begins to show a rectified component. In sum then we see that the addition of a third level and a second light field can significantly alter the nature of the light pressure force producing rectification and/or vortex forces. Moreover, we show that under certain conditions there is a direct connection of the stimulated vortex force to the rectified dipole force, because they both derive from the stimulated redistribution of photons among the modes of the exciting field.

\section{Summary}

We have presented several different results which demonstrate the point that the light pressure force for more than one dimension, more than two-level and more than one field frequency can differ substantially from the simpler one-dimensional, two-level, one monochromatic field case. These differences can give rise to striking new effects which are crucial in the understanding and application of laser cooled and trapped atoms.

\section{Acknowledgments}

N.P.B. would like to thank the organizers for the invitation to present this paper at the conference on Quantum Optics III. N.P.B. is an Alfred P. Sloan Research Fellow. The experimental work in Sec. 5 was performed in collaboration with M.G. Prentiss, P.R. IIemmer, M.S. Shahriar, D. Katz and J. Mervis. 


\section{References}

[1] See for example the special issues of JOSA B, November 1985 and 1989.

[2] J. Dalibard, C. Cohen-Tannoudji, J. Opt. Soc. Am. 11, 1707 (1985).

[3] J. Dalibard, C. Salomon, A. Aspect, E. Arimondo, R. Kaiser, N. Vansteenkiste, C. Cohen-Tannoudji, in: Proc. 11th Internat. Conf. Atomic Phys., Eds. Harosche, Gay, Grynberg, World Scientific, New Jersey 1989.

[4] S. Chu, D.S. Weiss, Y. Shevy, P.J. Ungar, in: Proc. 11th Internat. Conf. Atomic Phys., Eds. Harosche, Gay, Grynberg, World Scientific, New Jersey 1989.

[5] A. Aspect, J. Dalibard, A. Heidmann, C. Salomon, C. Cohen-Tannoudji, Phys. Rev. Lelt. 57, 1688 (1986).

[6] P.D. Lett, R.N. Watts, C.I. Westbrook, W.D. Phillips, P.L. Gould, H.J. Metcalf, Phys. Rev. Lett. 61, 169 (1988).

[7] J.P. Gordon, A. Ashkin, Phys. Rev. A 21, 1606 (1980).

[8] A. Ashkin, Phys. Rev. Lett. 40, 729 (1978).

[9] R. Grimm, Yu.B. Ovchnnikov, A.I. Sidorov, V.S. Letokhov, Phys. Rev. Lett. 65, 1415 (1990).

[10] A.P. Kazantsev, I.V. Krasnov, J. Opt. Soc. Am. B 6, 2140 (1989).

[11] V.S. Voitsekhovich, M.V. Danileiko, A.M. Negrijko, V.I. Romanienko, L.P. Yatsenko, Sov. Phys. Tech. Phys. 33, 690 (1988).

[12] J. Javanainen, Phys. Rev. Lett. 64, 519 (1990).

[13] A.P. Kazantsev, I.V. Krasnov, Sov. Phys. JETP Lett. 46, 332 (1988).

[14] A. Hemmerich, T.W. Hänsch, Phys. Rev. Lett. 68, 1492 (1992).

[15] K. Molmer, K. Berg-Sorensen, E. Bonderup, J. Phys, B 24, 2327 (1991).

[16] A. Aspect, E. Arimondo, R. Kaiser, N. Vansteenkiste, C. Cohen-Tannoudji, Phys. Rev. Lett. 61, 826 (1988).

[17] H.J. Gray, R.M. Whitley, C.R. Stroud, Jr., Optics Lett. 3, 218 (1978).

[18] M.G. Prentiss, N.P. Bigelow, M.S. Shahriar, P.R. Hemmer, Opt. Lett. 16, 1695 (1991).

[19] P.R. Hemmer, M.G. Prentiss, M.S. Shahriar, N.P. Bigelow, Opt. Commun. 89, 335 (1992).

[20] A.I. Sidorov, R. Grimm, V.S. Letokhov, J. Phys. B 24, 3733 (1991).

[21] P.R. Hemmer, M.S. Shahriar, M.G. Prentiss, D.P. Katz, K. Berggren, J. Mervis, N.P. Bigelow, Phys. Rev. Lett. 68, 3148 (1992).

[22] T. Cai, N.P. Bigelow, Optics Comm. 104, 175 (1993).

[23] M.S. Shahriar, P.R. Hemmer, M.G. Prentiss, P. Marte, J. Mervis, D. P. Katz, T. Cai, N.P. Bigelow, to be published, Phys. Rev. A Rapid Comm. 